\title{
La necessità di competenze multidisciplinari e il valore aggiunto della Cardiologia Riabilitativa nel paziente cardioperato
}

\author{
Pier Luigi Temporelli
}

Monaldi Arch Chest Dis 2006; 66: 136-138.

Divisione di Cardiologia Riabilitativa, Fondazione Salvatore Maugeri, IRCCS, Centro Medico di Veruno (NO); E-mail: ptemporelli@fsm.it

Dal censimento delle strutture riabilitative del 2005 emerge che più del $50 \%$ dei pazienti che afferiscono alle strutture di Cardiologia Riabilitativa sono cardioperati. Anche per il paziente cardiochirurgico, come in generale per il cardiopatico, l'età sta crescendo in maniera vertiginosa $\mathrm{e}$, in particolare, sta salendo la percentuale di ultrasettantacinquenni, pazienti fragili, disabili, decondizionati. Nella figura 1 è riportata la percentuale di cardioperati anziani (>75 anni) afferiti alle strutture di Cardiologia Riabilitativa della Fondazione Maugeri negli ultimi 10 anni, che conferma il trend in rapida crescita. Al contrario dell'età, la frazione d'eiezione del cardioperato si sta riducendo in maniera altrettanto vertiginosa: secondo i registri americani siamo passati da una frazione d'eiezione media del $65 \%$ negli anni '80 ad un 40\% nel 2005. Alla luce di queste premesse, siamo in pratica passati da una tipologia di paziente degli anni ' 80 che probabilmente oggi potrebbe essere inviato a domicilio e gestito ambulatorialmente, ad un tipo di paziente che assolutamente necessita di un ulteriore ricovero in struttura protetta dopo la fase acuta post-chirurgica. In realtà, che i problemi potrebbero esserci anche in pazienti apparentemente non complicati emerge in tutti i database delle cardiochirurgie Americane, che non hanno strutture di appoggio riabilitative. Da una recente esperienza di un singolo centro [1], a poca distanza dalla dimissione (<30 giorni) una consistente percentuale di pazienti è stata ri-ospedalizzata per complicanze di vario tipo quali infezioni, scompenso, ischemia, aritmie, embolia polmonare ed altro ancora. Mutuando per la realtà italiana dal nostro database di Fondazione Maugeri di Veruno, a cui che afferiscono circa 900 cardioperati/anno provenienti da diverse cardiochirurgie del nord-ovest, emergono sostanzialmente le stesse frequenti complicanze, con al primo posto per incidenza le tachiaritmie atriali. La fibrillazione atriale è una complicanza frequente, apparentemente banale ma in grado di condizionare la durata della degenza sia nella fase immediatamente post-operatoria che in quella tardiva e, soprattutto, di aumentare l'assorbimento di risorse [2]. Dal punto di vista fisiopatologico molti fattori condizionano l'insorgenza di fibrillazione atriale nel cardioperato; su tutti l'ipertono simpatico. Per tale motivo le Linee Guida raccomandano il betabloccante per tutti i pazienti che vanno incontro a cardiochirurgia, salvo controindicazioni assolute; in pazienti a rischio elevato pre-operatorio l'associazione di Amiodarone in fase peri-operatoria è giustificata [3]. Purtroppo, a dispetto delle chiare indicazioni in letteratura l'utilizzo del betabloccante in fase peri-operatoria e post-operatoria è largamente inadeguato (4].

Una complicanza spesso misconosciuta e sottostimata perché difficile da individuare nei pazienti

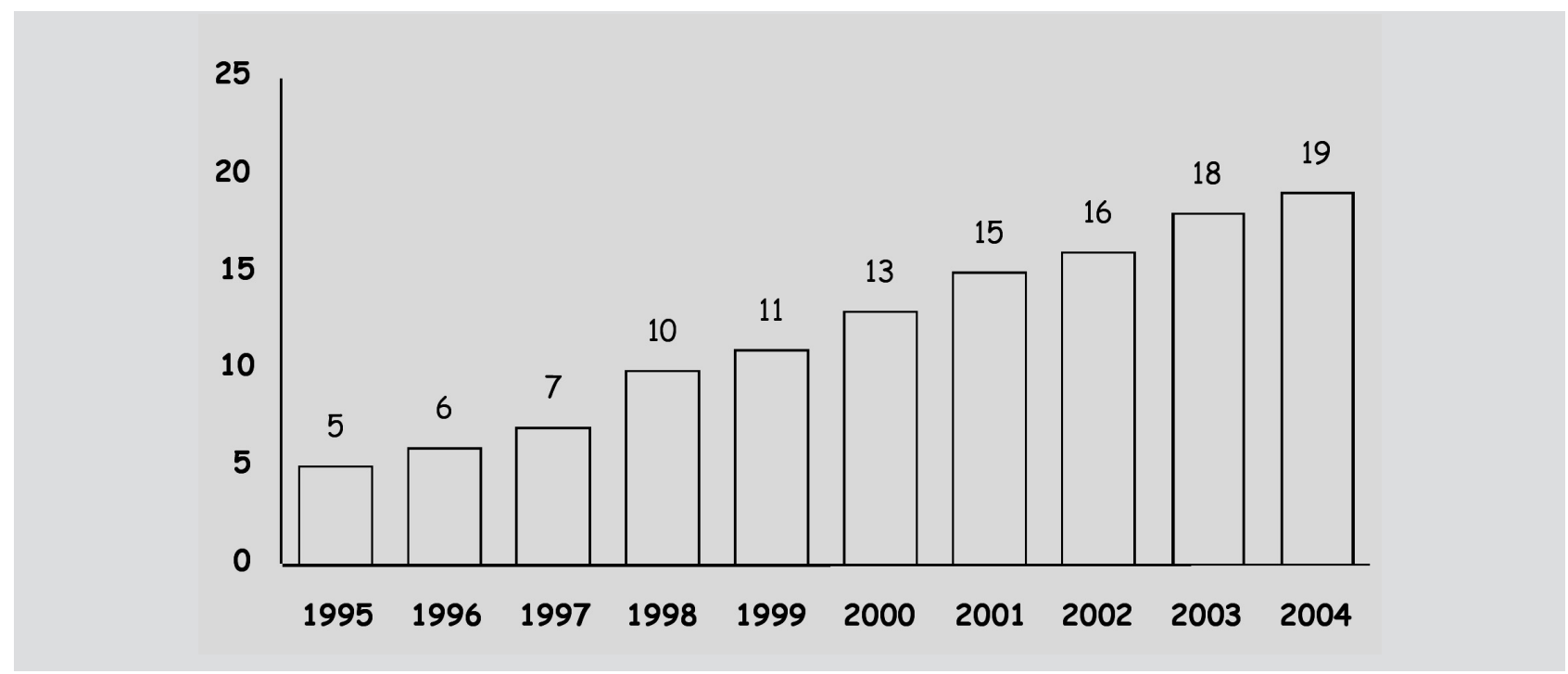

Figura 1. - Percentuale di cardioperati anziani (>75 anni) afferiti alle strutture di Cardiologia Riabilitativa della Fondazione Salvatore Maugeri nel decennio 1995-2004 (su un totale di 64582 pazienti). 
pauci/asintomatici è l'embolia polmonare, che può presentarsi anche in soggetti apparentemente a basso rischio. Siccome molte embolie non vengono diagnosticate, è verosimile che l'incidenza nel cardioperato recente non sia solo $1-2 \%$, come riportato in letteratura, anche perché è stato dimostrato [5] che l'incidenza di trombosi venose profonde negli stessi pazienti è ben più elevata (circa 17\%). Inoltre, è stato recentemente dimostrato che l'uso della tromboprofilassi dopo bypass, e peggio dopo sostituzione valvolare meccanica, è tutt'altro che ottimale, favorendo pertanto la probabilità di fenomeni embolici.

Un problema numericamente contenuto ma importante dal punto di vista clinico è quello delle infezioni nel post-operatorio: la mediastinite, ad esempio è una temibile complicanza che allunga sensibilmente la durata della degenza, i costi e, problema più grave, la mortalità. Per tale motivo nel paziente a rischio (diabetico, obeso, settico, ri-operato) è necessario seguire sia in fase acuta che post-acuta una flow chart ben precisa, che prevede l'indispensabile monitoraggio clinico, le colture seriate, la terapia antibiotica standardizzata, le medicazioni standardizzate. In questo contesto, un atteggiamento preventivo dimostratosi efficace è lo screening dei portatori di stafilococco aureo a livello nasale. È stato infatti dimostrato che la bonifica con un semplice antibiotico locale dei portatori a livello nasale di stafilococco aureo meticillino-resistente è in grado di ridurre l'incidenza di infezioni nel post-operatorio [6].

Un altro problema clinico sempre più frequente è quello del bypassato disfunzionante, popolazione in continua crescita. Da studi in letteratura sappiamo che in pazienti trivasali con funzione sistolica depressa l'atteggiamento chirurgico è da preferirsi a quello conservativo o a quello interventistico meccanico. Una delle possibili motivazioni potrebbe essere l'effetto favorevole dell'intervento sul rimodellamento ventricolare. Nella nostra esperienza abbiamo studiato il fenomeno del rimodellamento in un consistente gruppo consecutivo di pazienti rivascolarizzati e disfunzionanti ( $\mathrm{FE}<35 \%$ ) dopo bypass. Non del tutto atteso, abbiamo trovato che a soli 6 mesi dall'intervento un terzo di questi pazienti "rimodella" in maniera sfavorevole. Di rilievo, nei pazienti che hanno rimodellato l'incidenza di morte cardiaca, scompenso e reinfarto è 3 volte superiore a quella dei pazienti che non hanno rimodellato. All'analisi multivariata, assieme alla classe funzionale NYHA all'arrivo in cardiologia riabilitativa il rimodellamento a sei mesi è emerso come il più potente predittore di eventi nel follow-up. Alla luce di questa esperienza il nostro atteggiamento in questi pazienti è diventato più aggressivo in termini di terapia e controlli.

Molte altre sono le possibili complicanze, da quelle neurologiche a quelle renali, la cui completa trattazione non è l'obiettivo di questo capitolo. Il concetto invece da sottolineare è che alla luce della diversa natura della complicanze l'approccio al cardioperato deve essere multidisciplinare e riassume ciò che oggi la Cardiologia Riabilitativa intende per riabilitazione omnicomprensiva e multidimensionale del cardiopatico, in questo caso del cardioperato. L'interazione con neurologo, pneumologo, infettivo$\log 0$, nefrologo e fisiatra è sempre più mandatoria; nello stesso tempo, più figure mediche e paramediche quali infermieri, fisioterapisti, terapisti della riabilitazione, dietisti e psicologi devono operare a stretto contatto per un intervento più efficace e duraturo.

È per questo che la riabilitazione del paziente cardioperato è una "straordinaria occasione clinica"; permette innanzitutto di fare assistenza medica in termini di riconoscimento precoce e trattamento delle complicanze post-chirurgiche. Ma oltre alla assistenza, anche per il cardioperato la Cardiologia Riabilitativa prevede il recupero funzionale e la stratificazione prognostica. Nonostante il cardioperato sia sempre più anziano con disabilità e comorbidità è ancora possibile impostare nella stragrande maggioranza dei casi un programma di training fisico che deve concludersi, specie nel cardiopatico ischemico, con una valutazione funzionale anche sottomassimale. La prova ergometrica in fase precoce serve infatti non tanto e solo per la ricerca di ischemia quanto piuttosto per valutare la capacità lavorativa. Anche nel cardioperato infatti, la capacità lavorativa è un potente predittore di prognosi: da una esperienza francese su più di 2500 pazienti una durata di esercizio $>7$ minuti, in media a 30 giorni dopo bypass, identifica i pazienti con prognosi più favorevole [7].

Infine, una peculiarità del setting riabilitativo nel cardioperato coronaropatico è l'impostazione di un programma di educazione sanitaria e prevenzione secondaria. Non dobbiamo dimenticare che il processo dell'aterosclerosi non si cura solo con il bypass, con l'angioplastica o con lo stent, ma anche intervenendo e modificando i fattori di rischio convenzionali in modo da modificare efficacemente la storia naturale della malattia. Tra l'altro, sappiamo che nel followup del cardioperato molti farmaci "salvavita" vengono spesso precocemente abbandonati. In una esperienza australiana su 2500 pazienti, a 4 anni in media dal bypass solo una modesta percentuale assumeva ancora regolarmente anti-aggreganti, statine, ACEinibitori, o betabloccanti [8]. In ogni caso, aldilà dell'inconfutabile effetto dei farmaci, un profilo di vita scorretto è in grado di condizionare sfavorevolmente la prognosi anche nel cardioperato. Ad esempio, è stato dimostrato come anche dopo bypass smettere di fumare garantisce una sopravvivenza nel lungo termine decisamente migliore rispetto a coloro che continuano a fumare [9].

\section{Conclusioni}

Grazie alle competenze acquisite nel corso degli ultimi 20 anni ed ai modelli organizzativi multidisciplinari sviluppati, la Cardiologia Riabilitativa oggi costituisce una straordinaria occasione clinica e un indiscutibile plus-valore nella gestione del paziente cardioperato.

\section{Bibliografia}

1. Hannan EL, Racz MJ, Walford G, et al. Predictors of Readmission for Complications of Coronary Artery Bypass Graft Surgery. JAMA 2003; 290: 773-780.

2. Mathew JP, Fontes ML, Tudor IC, et al. A Multicenter Risk Index for Atrial Fibrillation After Cardiac Surgery. JAMA 2004; 291: 1720-1729. 
3. ACC/AHA Guidelines for coronary artery bypass graft surgery: Executive summary and recommendations. Circulation 1999; 100: 1464-1480.

4. Ferguson TB Jr, Coombs LP, Peterson ED, et al for the Society of Thoracic Surgeons National Adult Cardiac Surgery Database. Preoperative Beta-Blocker Use and Mortality and Morbidity Following CABG Surgery in North America. JAMA 2002; 287: 2221-2227.

5. Ambrosetti M, Salerno M, Zambelli M, et al. Deep vein thrombosis among patients entering cardiac rehabilitation after coronary artery bypass surgery. Chest 2004; 125: 191-6.

6. Perl TM, Cullen JJ, Wenzel RP, et al. Intranasal Mupirocin to prevent postoperative Staphylococcus Aureus infections. N Engl J Med 2002; 346: 1871-7.
7. Sellier P, Chatellier G, D'Agrosa-Boiteux MC, et al. Use of non-invasive cardiac investigations to predict clinical endpoints after coronary bypass graft surgery in coronary artery disease patients: results from the prognosis and evaluation of risk in the coronary operated patient (PERISCOP) study. Eur Heart J 2003; 24: 916-26.

8. Bradshaw PJ, Jamrozik K, Gilfillan I, Thompson PL. Preventing recurrent events long term after coronary artery bypass graft: suboptimal use of medications in a population study. Am Heart J 2004; 147: 1047-53.

9. van Domburg RT, Meeter K, van Berkel DF, et al. Smoking cessation reduces mortality after coronary artery bypass surgery: a 20-year follow-up study. $J$ Am Coll Cardiol 2000; 36: 878-83. 